\author{
Marquette University \\ e-Publications@Marquette
}

$9-2013$

\title{
Automated Multi-Objective Design Optimization of PM AC Machines Using Computationally Efficient FEA and Differential Evolution
}

\author{
Gennadi Y. Sizov \\ Marquette University \\ Peng Zhang \\ Marquette University \\ Dan M. Ionel \\ University of Wisconsin - Milwaukee \\ Nabeel Demerdash \\ Marquette University, nabeel.demerdash@marquette.edu \\ Marius Rosu \\ ANSYS Inc.
}

Follow this and additional works at: https://epublications.marquette.edu/electric_fac

Part of the Computer Engineering Commons, and the Electrical and Computer Engineering Commons

\section{Recommended Citation}

Sizov, Gennadi Y.; Zhang, Peng; Ionel, Dan M.; Demerdash, Nabeel; and Rosu, Marius, "Automated MultiObjective Design Optimization of PM AC Machines Using Computationally Efficient FEA and Differential Evolution" (2013). Electrical and Computer Engineering Faculty Research and Publications. 203.

https://epublications.marquette.edu/electric_fac/203 
Marquette University

e-Publications@Marquette

\section{Electrical and Computer Engineering Faculty Research and Publications/College of Engineering}

This paper is NOT THE PUBLISHED VERSION.

Access the published version at the link in the citation below.

IEEE Transactions on Industry Applications, Vol. 49, No. 5 (September-October 2013): 2086-2096. DOI. This article is (C) The Institute of Electrical and Electronics Engineers and permission has been granted for this version to appear in e-Publications@Marquette. The Institute of Electrical and Electronics Engineers does not grant permission for this article to be further copied/distributed or hosted elsewhere without the express permission from The Institute of Electrical and Electronics Engineers.

\section{Automated Multi-Objective Design Optimization of PM AC Machines Using Computationally Efficient FEA and Differential Evolution}

Gennadi Y. Sizov

Department of Electrical and Computer Engineering, Marquette University, Milwaukee, WI Peng Zhang

Department of Electrical and Computer Engineering, Marquette University, Milwaukee, WI Dan M. Ionel

Department of Electrical and Computer Engineering, Marquette University, Milwaukee, WI Nabeel A. O. Demerdash

Department of Electrical and Computer Engineering, Marquette University, Milwaukee, WI 


\title{
Marius Rosu
}

ANSYS, Inc., Pittsburgh, PA

\begin{abstract}
:
The design optimization methods described in this paper are employing an ultrafast computationally efficient finite element analysis technique. A minimum number of magnetostatic solutions are used for the analysis, which makes possible the study of thousands of candidate motor designs with typical PCworkstation computational resources. A multi-objective differential evolution algorithm that considers a large number of independent stator and rotor geometric variables and performance criteria, such as average and ripple torque, losses, and efficiency, is used. The optimization method is demonstrated on different permanent magnet (PM) ac synchronous motors in the kilowatt and megawatt power ranges. For the low-power PM ac machine study, a nine-slot six-pole topology is considered. For the highpower PM ac machines, four case studies were carried out with the following: fractional-slot embedded surface PM (SPM), fractional-slot interior PM (IPM), integer-slot SPM, and integer-slot IPM, respectively. Four motor topologies are systematically compared based on optimal Pareto sets. The design optimization of IPM motors includes an additional search for an optimum operating torque angle corresponding to the maximum-torque-per-ampere condition.
\end{abstract}

\section{SECTION I. Introduction}

The design of electric machinery is complicated by the usually large number of design variables related to complex geometries and material properties. In addition, the designer is often confronted by a large number of conflicting design objectives that further complicate the design process. In a typical design flow, initial device sizing is performed using analytical and magnetic equivalent circuit models, and finite element analysis (FEA) is used in the final steps of the design process for verification and parameter fine-tuning. In this approach, several iterations may be performed to satisfy a limited number of objectives. Furthermore, a majority of the design variables are predetermined based on the analytically derived models, and then, a small number of variables are fine-tuned using FEA. In this approach, only a small number of variables are varied using FEA, and the optimality of the final design is not guaranteed. On the other hand, a design process that relies solely on conventional FEA models to investigate a large number of candidate designs may be inadequate due to the prohibitively long computer execution times. This limitation of FEA-based models has prevented their application in large-scale design optimization studies, where a significant number of design parameters are varied in the search for optimum designs.

Recent developments in the area of FEA-based evolutionary design synthesis and optimization were reviewed by Duan and lonel [1] and can be classified into two distinct classes, the methods that consider complete transient solutions and rely on advanced computing facilities proposed by Jiang et al. [2] and the methods that rely on reduced-effort models in order to estimate machine performance during the iterative search. Examples of the latter include the work of Parasiliti et al. [3], Pellegrino and Cupertino [4], Arkadan et al. [5], Giurgea et al. [6], Sarikhani and Mohammed [7], and Ouyang et al. [8]. 
Several reduced-effort FEA modeling approaches have been proposed in the literature, e.g., [9][10][11][12][13][14][15]. These techniques aim to minimize the computational effort associated with FEA while maximizing the amount of available machine performance information. Previous methods were able to estimate the average torque and the torque ripple [9]-[10][11] or the stator core losses [12]. Computationally efficient FEA (CE-FEA) was introduced by the authors in [13] and [14]. In this new approach, both magnetic and electric circuit symmetries of permanent-magnet (PM) ac machines were exploited to reduce the number of required static finite element (FE) solutions. In CEFEA, a comprehensive set of performance indices, including the induced voltage waveforms, average torque, profiles of cogging torque and torque ripple, flux density waveforms, winding inductances, and core losses, is extracted from a minimum number of magnetostatic FE solutions with a machine representation in the abc reference frame. The accuracy of such an approach was validated by results from time-stepping (transient) FEA (TSFEA) [14], [16].

In this paper, an automated design tool that couples the CE-FEA computational method and the differential evolution (DE) optimizer [17] was developed. The method is demonstrated on two comprehensive large-scale design optimization studies that involve the evaluation of thousands of candidate designs. The first design study shows the application of the automated tool to the optimization of the existing design. This study demonstrates the possibility of torque-dense and torque-ripple-free design of the interior PM (IPM) motor with three slots per pole pair. The second design study shows the application of the method to the systematic motor technology comparison where four high-power motor topologies are compared based on the optimal Pareto sets. The following four case-study machines are optimized and compared based on optimal Pareto sets: fractional-slot embedded surface PM (SPM), fractional-slot IPM (F-IPM), integer-slot embedded SPM, and integer-slot IPM (I-IPM). In both examples, a large number of stator and rotor variables are used to automatically optimize the average torque, the efficiency, and the cogging and the ripple torques.

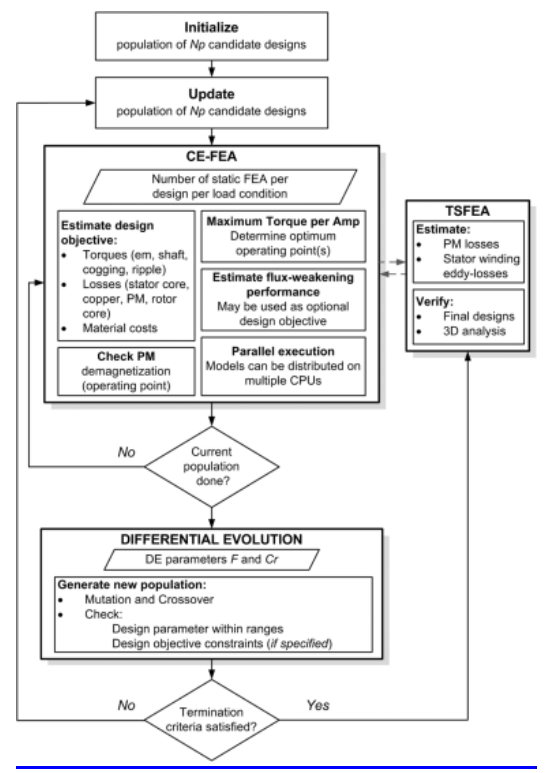

Fig. 1. Block diagram for model-based design optimization procedure employing CE-FEA and DE optimizer.

The block diagram of the proposed model-based design optimization is shown in Fig. 1. In this figure, a DE algorithm is used to dynamically update and improve the candidate design population, and the CE- 
FEA is used to extract the objectives (average and ripple torques, core losses, etc.). It should be noted that, for the case study, IPM machine parameters, such as the average electromagnetic and shaft torques, torque ripples, and efficiency, are extracted at a single load point from several magnetostatic solutions. These solutions take several seconds to execute using a typical PC-based workstation.

\section{SECTION II. Ce-Fea}

The CE-FEA is applicable to the steady-state analysis of sine-wave energized current-regulated synchronous machines. In CE-FEA, a static nonlinear finite-element solver is utilized, and significant computational savings are obtained by utilizing additional symmetries of the magnetic circuit (beyond odd/even periodicity conditions) and electric circuits. The machine is analyzed/evaluated at several rotor positions with corresponding sequentially sampled phase current excitations based on the sinusoidal current waveform. Outputs such as the magnetic vector potentials, phase flux linkages, and stator core flux densities are reconstructed using the waveform symmetry conditions that stem from the magnetic and electric circuit symmetries. The corresponding Fourier series of the reconstructed quantities are obtained paying special attention to possible aliasing effects [14]. As a result, using the CE-FEA, performance parameters such as the back-EMF waveforms, average torque, profiles of cogging torque and torque ripples, and stator core losses can be extracted from a minimum number of magnetostatic FE solutions. This leads to a significant reduction of execution times when compared to the laborious TSFEA approaches while preserving the main benefits of FE solvers, i.e., accuracy of geometric shape and magnetic saturation representations. For example, to evaluate a candidate motor at a single operating condition, CE-FEA requires as few as three magnetostatic solutions (three rotor positions) for average torque and six solutions (six rotor positions) for detailed analysis that includes terminal voltage, torque ripple, and accurate core loss calculation [14].

In the present CE-FEA framework, additional loss components can be estimated using a combination of analytical and numerical methods. Eddy-current induced PM losses [18] and stator conductor induced eddy-current losses [19], [20] can be accounted for by means of analytical and/or numerical models. Meanwhile, the core losses and PM losses caused by the inverter/drive switching can be implemented into the CE-FEA utilizing the method presented in [21]. In the block diagram of the present modelbased optimization procedure, the TSFEA can be used to estimate the eddy-current induced losses in both PMs and stator coils. After optimal designs are selected, a detailed TSFE analysis that accounts for additional loss components due to the inverter switching, not accounted for in the CE-FEA, should be performed. Further TSFE analysis may include the simulation of the effects of induced eddy currents in the lamination steel on the overall field distribution in the machine, as discussed in [22]. In addition, TSFEA-based numerical models can be used in selecting the appropriate circumferential and axial PM segmentations that will minimize induced eddy-current magnet losses. 


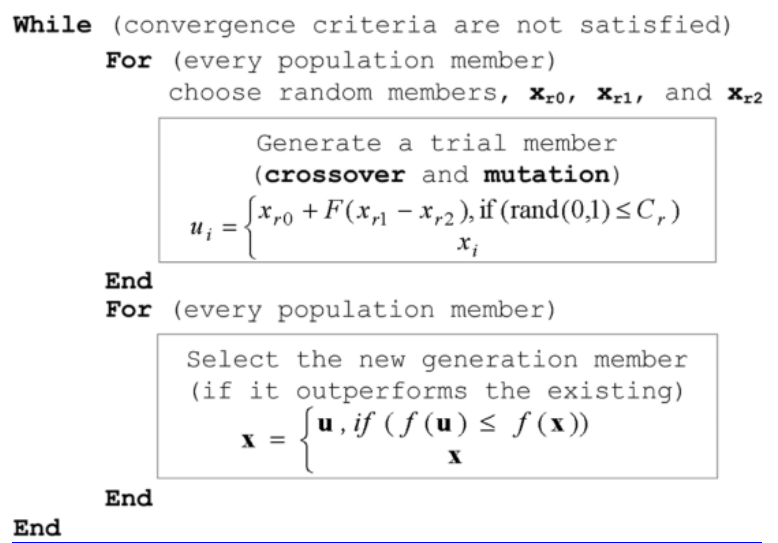

Fig. 2. Simplified pseudocode of DE implementation for a single-objective minimization problem.

\section{SECTION III. Design Optimization}

\section{A. Optimum Operating Point (MTPA)}

The design optimization of IPM machines is complicated by the fact that the maximum torque production is a function of the advance angle of the current, which, in turn, is a function of the design parameters. This means that, for a systematic comparison of candidate designs, an additional search/optimization of the optimum operating point [maximum torque per ampere (MTPA)] has to be performed for every candidate design. The determination of the MTPA operating condition requires additional model iterations to obtain the optimum operating condition, hence increasing the execution time. This, however, is not of significant concern with CE-FEA, where several static FE solutions are sufficient for the estimation of the average torque [14].

\section{B. DE Optimization Algorithm}

The DE algorithm belongs to the wide class of metaheuristic optimizers that attempts to find a global minimum/maximum by iteratively improving a population of candidate designs until the convergence criteria are satisfied [17]. The differential part of the DE algorithm implies that, unlike other derivativefree population-based evolutionary algorithms (genetic algorithms, particle swarms, simulated annealing, etc.), DE utilizes a weighted difference between candidate designs to facilitate the improvement of future generations. The DE approach has been shown to outperform most populationbased evolutionary algorithms on a number of benchmark test functions [1], [17].

In DE's most basic form (see Fig. 2), the parameters $u_{i}$ of the new trial member $\mathbf{u}$ are updated based on mutation and crossover ideas given in the following:

$$
u_{i}= \begin{cases}{\left[x_{r 0}+F\left(x_{r 1}-x_{r 2}\right)\right]} & \text { if }(\operatorname{rand}(0,1)) \leq C_{r} \\ x_{i} & \end{cases}
$$

where $x_{i}$ is the parameter of the present population member and $\left[x_{r 0}+F\left(x_{r 1}-x_{r 2}\right)\right]$ is the mutation operation applied to the parameters of the three randomly selected present population members, $x_{r 0}, x_{r 1}$, and $x_{r 2}$, with $\mathrm{F}$ being a positive real difference scale factor. The mutation operation 
is carried out only if the following crossover condition is satisfied: rand $(0,1) \leq C_{r}$, where $C_{r}$ is a predefined crossover probability. Once the trial member $\mathbf{u}$ is created, its objective function $f(\mathbf{u})$ is evaluated and compared to the objective function of the present member $f(\mathbf{x})$. The trial vector $u$ is allowed to enter the population only if it outperforms the present member $\mathbf{x}$. The population size is heuristically chosen to be ten times the number of design variables [17].

There are two approaches to multi-objective optimization. The most straightforward approach is based on a weighted sum of objectives that transforms a multi-objective problem into a simple singleobjective case, as given in

$$
f_{1}=\sum_{n=1}^{N} w_{n} f_{n}(\mathbf{x})
$$

(2)

However, in this approach, a choice of weights $w_{n}$ may have a significant impact on the optimality of the final design. Moreover, the dual nature of some objectives (conflicting/nonconflicting) may lead to problems with proper weight assignment. For a given set of weights, this approach leads to a single optimal design that either maximizes or minimizes the objective function given in (2). The second approach to multi-objective optimization problems, used in this work, is based on the Paretodominance selection criteria. This approach typically results in the family of the best compromise designs that provide the designer with a clear view of various tradeoffs between a number of Paretooptimal designs. Pareto-dominance selection criteria can be incorporated into the DE using several approaches outlined in [17] and [23].

\section{Problem Statement}

The multi-objective definition of the PM ac machine design used in this paper can be summarized as follows:

- minimize torque ripple and total losses (core and copper), while

- maximizing the torque production per unit volume at rated load.

One way to achieve this is by considering two objectives given by the following:

$$
\begin{aligned}
& \text { minimize: } f_{1}=T_{e m(p k-p k)}, \\
& \text { maximize: } f_{2}=\frac{T_{e m}}{\sqrt{P_{C u}+P_{F e}}}
\end{aligned}
$$

where $f_{1}$ corresponds to the peak-to-peak torque ripple and $f_{2}$ corresponds to "goodness," which is a measure of average torque production with respect to total losses. The outside diameter and axial length are fixed during the optimization. The axial length is then scaled to achieve the desired output ratings of different optimized machine designs. The current density and slot fill are fixed, respectively. 
Every candidate design for these IPM machines is evaluated at MTPA condition. The PM material and steel used in the core construction are fixed. A purely sinusoidal current-regulated sine-wave drive is assumed to be energizing such machines.

\section{SECTION IV. Case Study 1: Low-Power IPM Machine (9-Slot, 6-Pole)}

\section{A. Problem Statement}

In this section, an optimization study of the IPM machine with fractional-slot ( $q=$

0.5 slots/pole/phase) shown in Fig. 3 is discussed. As a preliminary step, the numerical analysis model was validated against experimental data for a prototype motor, which is referenced in the following as a "typical design." Satisfactory agreement was noted for various load conditions, as exemplified in Table I.

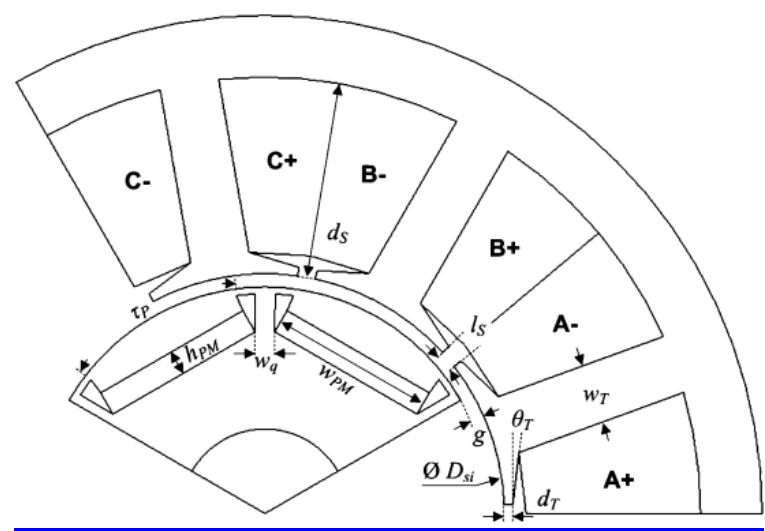

Fig. 3. Cross section of the 9-slot 6-pole IPM machine with 11 independent geometric variables used in multiobjective optimization.

Table I Experimental Results Used for Validation and Calibration of the Computational Model

\begin{tabular}{|l|l|l|l|l|l|}
\hline Speed & & Torque $[\mathrm{Nm}]$ & Current $\left[\mathrm{A}_{r m s}\right]$ & Copper Loss [W] & Core Loss [W] \\
\hline $1800 \mathrm{r} / \mathrm{min}$ & Exp. & 7.4 & 8.1 & 49.9 & 49.6 \\
\hline & Calc. & 7.5 & 8.1 & 50.9 & 45.9 \\
\hline & Exp. & 14.7 & 15.7 & 187.4 & 55.4 \\
\hline & Calc. & 15.0 & 15.7 & 191.2 & 52.2 \\
\hline $3600 \mathrm{r} / \mathrm{min}$ & Exp. & 7.4 & 8.6 & 56.3 & 147.6 \\
\hline & Calc. & 7.5 & 8.6 & 57.4 & 140.8 \\
\hline & Exp. & 14.6 & 16.3 & 201.9 & 178.1 \\
\hline & Calc. & 15.0 & 16.3 & 206.1 & 167.2 \\
\hline
\end{tabular}

The machine has been optimized following the procedure outlined in Sections II and III, with design parameter limits set to the values provided in Table II. The population size of a single generation was set to 100 , and the DE algorithm was executed for 100 generations. These settings resulted in the total of 10000 candidate design evaluations. In the CE-FEA, six magnetostatic FE solutions are used to perform a detailed evaluation of every candidate motor operating at MTPA load condition. Additional search for MTPA operating condition is performed on every candidate design requiring four additional reduced CE-FEA evaluations with three static solutions each. Reduced CE-FEA with three static 
solutions per torque (advance) angle allows to rapidly estimate average torque [14] while ignoring torque ripple and bypassing costly post-processing associated with flux density computations that require additional computational effort. The total simulation time required for the evaluation of 10000 candidate designs on a single core of the PC-based workstation is approximately $52 \mathrm{~h}$. The detailed breakdown of model simulation times is provided in Table III.

Table II Optimization Parameters: Limits and Values Corresponding to Machine of Fig. 5. Values are Per Unit of Pole-Pitch Arc Length, and the Unit for Angle Is Mechanical Degree

\begin{tabular}{|l|c|r|r|r|r|l|l|}
\hline $\begin{array}{l}\text { Optimized } \\
\text { Parameters }\end{array}$ & $\begin{array}{l}\text { Fractional-slot (9-slot, 6- } \\
\text { pole) }\end{array}$ & & & & & & \\
\hline & & Limits & & & Machine & & \\
\hline 7-stator, 4-rotor & & Lower & Upper & M-1 & M-2 & M-3 & Typ. \\
\hline Stator & $D_{s i}$ & 1.940 & 2.156 & 1.941 & 1.941 & 1.965 & 1.979 \\
\hline & $g$ & 0.027 & 0.069 & 0.028 & 0.040 & 0.062 & 0.034 \\
\hline & $w_{T}$ & 0.213 & 0.355 & 0.266 & 0.266 & 0.253 & 0.284 \\
\hline & $l_{s}$ & 0.062 & 0.275 & 0.075 & 0.070 & 0.084 & 0.156 \\
\hline & $d_{s}$ & 0.550 & 0.824 & 0.801 & 0.820 & 0.815 & 0.742 \\
\hline & $d_{T}$ & 0.029 & 0.048 & 0.028 & 0.036 & 0.036 & 0.038 \\
\hline & $\theta_{T}$ & 7.50 & 30.00 & 9.02 & 8.83 & 12.42 & 18.75 \\
\hline Rotor & $w_{P M}$ & 0.550 & 0.687 & 0.661 & 0.683 & 0.686 & 0.687 \\
\hline & $h_{P M}$ & 0.055 & 0.103 & 0.099 & 0.102 & 0.103 & 0.079 \\
\hline & $\tau_{P}$ & 0.548 & 0.940 & 0.641 & 0.627 & 0.600 & 0.778 \\
\hline & $w_{q}$ & 0.027 & 0.096 & 0.092 & 0.091 & 0.072 & 0.062 \\
\hline
\end{tabular}

Table III Number of Magnetostatic Solutions and Computational Time Required for Evaluation of a Single Candidate Design at MTPA

\begin{tabular}{|l|l|l|l|}
\hline CE-FEA & Solutions & Mesh density/Order & Computational Time $[\mathrm{s}]$ \\
\hline Reduced & 3 & $2384 \mathrm{el} . / 1^{\text {st }}$ & 2.2 \\
\hline Detailed & 6 & $2384 \mathrm{el} . / 2^{\text {nd }}$ & 10.1 \\
\hline TOTAL & $4 \times$ Reduced $+1 \times$ Detailed & & 18.9 \\
\hline
\end{tabular}

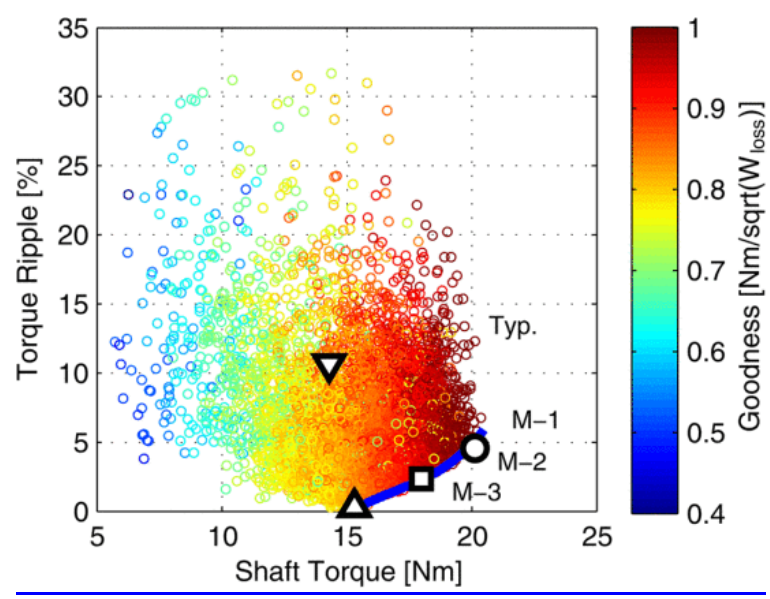

Fig. 4. Pareto-optimal set for design objectives. The three optimal machines $M-1, M-2$, and $M-3$ and the conventional machine are labeled. Shaft torque corresponds to the output torque after the core losses. 


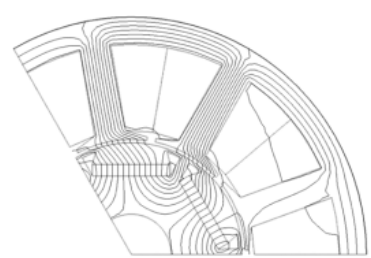

(a)

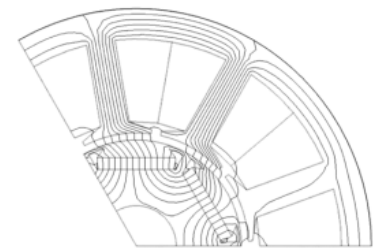

(c)

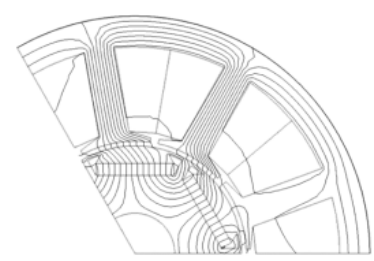

(b)

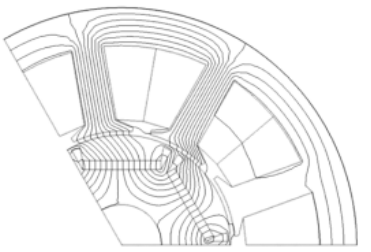

(d)

Fig. 5. Cross sections of optimized machines corresponding to Fig. 4 and Table II. Also shown are the flux plots for the rated-load conditions.

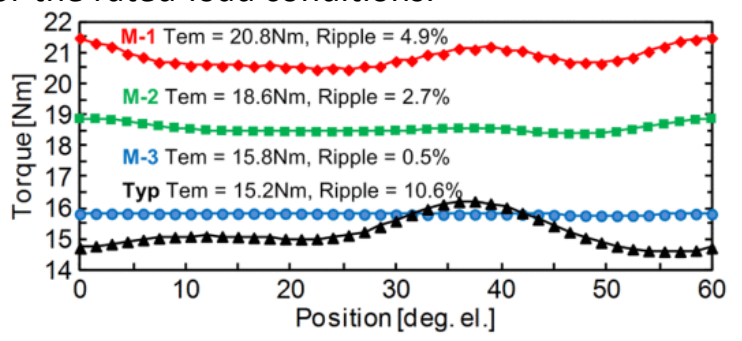

(a)

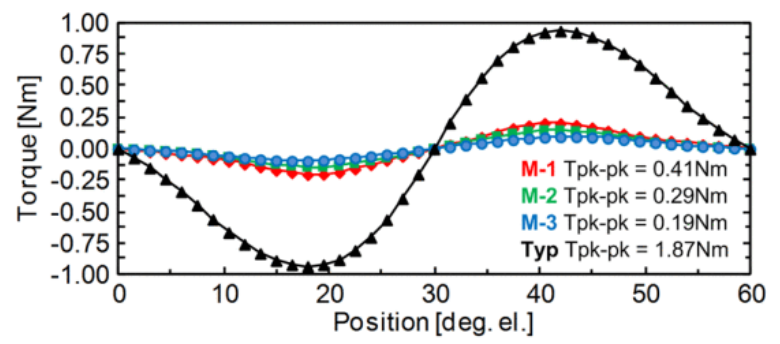

(b)

Fig. 6. Electromagnetic torque for machines of Fig. 5 (red: M-1; green: M-2; blue: M-3; black: typical design) supplied by a current-regulated sine-wave drive (purely sinusoidal currents are assumed). Torque profiles are verified/obtained using detailed FEA with Maxwell stress tensor and second-order elements. 

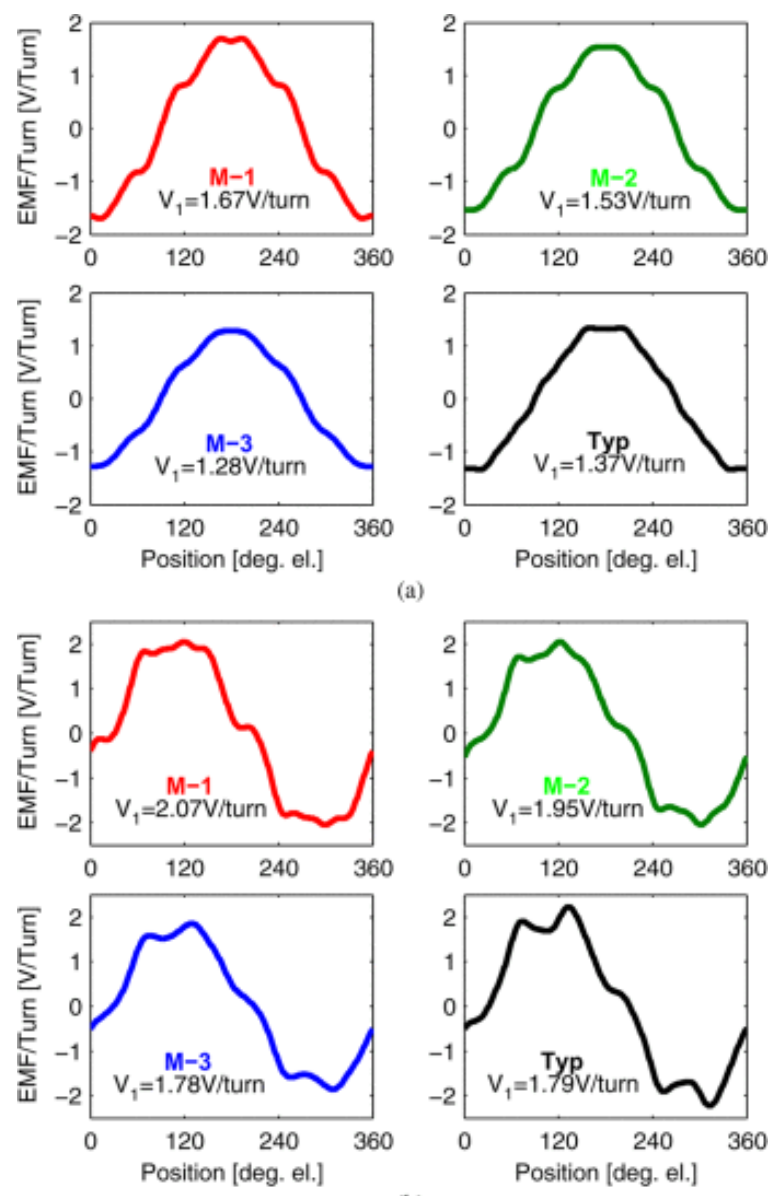

(b)

Fig. 7. Induced voltages at open-circuit and rated-load for machines in Fig. 5 (red: M-1; green: M-2; blue: M-3; black: typical design). Also shown are the peak values of the fundamental components.

Shown in Fig. 4 are the values of the design objectives specified above for 10000 candidate designs as well as the objective function values corresponding to the final Pareto-optimal set of designs. The average shaft torque that accounts for core losses is plotted in Fig. 4 to provide a familiar basis of comparison of average and ripple torque production. From Fig. 4, it should be observed that a large variation between the torque ripple and the average torque production occurs from values as low as $0.4 \%$ to $32 \%$, with the shaft torque varying from 6 to $21 \mathrm{~N} \cdot \mathrm{m}$. Three Pareto-optimal machines are selected from the Pareto-optimal set, where the candidate design $\mathrm{M}-1$ has the highest shaft torque (specific torque), the candidate design $\mathrm{M}-2$ is a compromise between high torque and low ripple, and M-3 poses the low torque ripple. Cross sections corresponding to machines $M-1, M-2$, and $M-3$ are shown in Fig. 5. Also shown in Fig. 5 is a typical design used for reference. The values of design variables corresponding to all machines are provided in Table II. Shown in Fig. 6(a) and (b) are the torque profiles corresponding to the four machines operating at rated-load (MTPA) and open-circuit (cogging torque) conditions, respectively. A significant reduction of both on-load and open-circuit ripple (cogging) is observed for the optimized designs. For machines $\mathrm{M}-1$ and $\mathrm{M}-2$, a significant increase in the average torque production is observed. More specifically, a $37 \%$ increase of the average torque is observed for machine M-1. From the results shown in Fig. 6(b), one observes a significant reduction of the cogging torque in the case of the optimized machines. The cogging torque is reduced by as much $90 \%$ in the case of the optimized machine $M-3$. It should be noted that results provided 
in Fig. 6 were verified with a detailed FEA that employs the Maxwell stress tensor and second-order finite elements for the calculation of the electromagnetic torque [16]. Open-circuit and rated-load induced voltages for the four machines are provided in Fig. 7, with their corresponding harmonic breakdowns given in Table IV.

\section{B. Design to Achieve Desired Ratings of $7.5 \mathrm{hp}(15 \mathrm{~N} \cdot \mathrm{m}$ and $3600 \mathrm{r} / \mathrm{min}$ )} Here, three optimized machines ( $M-1, M-2$, and $M-3$ ) and the typical (conventional) machine were scaled to achieve the desired 7.5-hp output rating. The axial length $l_{F e}$ is used to achieve this. Given in Table $V$ are the corresponding axial lengths in per unit (p.u.) of the pole-pitch arc length for the four machines. Also reported in Table $\mathrm{V}$ are the PM and total machine masses per unitized to the machine of typical proportions. As expected, machine M-1, having the highest specific torque [see Fig. 6(a)], results in the shortest and lightest machine that utilizes the least PM material for the given rating. More specifically, for machine $\mathrm{M}-1$, the mass of the PM material is reduced by $7 \%$ while the total machine mass and length are reduced by $25 \%$ and $23 \%$, respectively. This is while the rated-load torque ripple and open-circuit cogging torque are reduced by $54 \%$ and $79 \%$, respectively. This practically eliminates the need for stator and/or rotor skewing, hence reducing manufacturing costs. In the case of applications demanding higher torque production quality, further reductions of the torque ripple can be achieved with machines M-2 and M-3. Shown in Fig. 8 are the separations of loss components for the four machines. Here, it should be observed that machine M-2 results in the lowest overall loss and, hence, the highest efficiency of $94 \%$. An almost equal split between copper and core losses for machine M-2 should also be noted, which is another indication of the "optimality" of the design from a loss point of view. Overall, machine M-2 offers the best balance between the output torque quality, efficiency, and material and manufacturing costs.

Table IV Harmonic Analysis of Induced Voltages Shown in Fig. 7

\begin{tabular}{|l|l|l|l|l|l|l|}
\hline & Motors & Harmonic order & & & & \\
\hline & & $1^{\text {st }}$ & $5^{\text {th }}$ & $7^{\text {th }}$ & $11^{\text {th }}$ & $13^{\text {th }}$ \\
\hline Open circuit [V/turn] & $\mathrm{M}-1$ & 1.67 & 0.098 & 1.10 & 0.002 & 0.020 \\
\hline & $\mathrm{M}-2$ & 1.53 & 0.091 & 0.87 & 0.005 & 0.002 \\
\hline & $\mathrm{M}-3$ & 1.28 & 0.048 & 0.047 & 0.003 & 0.002 \\
\hline & Typ. & 1.37 & 0.014 & 0.046 & 0.012 & 0.022 \\
\hline & $\mathrm{M}-1$ & 2.07 & 0.245 & 0.131 & 0.020 & 0.032 \\
\hline & $\mathrm{M}-2$ & 1.95 & 0.185 & 0.124 & 0.034 & 0.024 \\
\hline & $\mathrm{M}-3$ & 1.78 & 0.194 & 0.059 & 0.021 & 0.004 \\
\hline & Typ. & 1.79 & 0.316 & 0.038 & 0.042 & 0.013 \\
\hline
\end{tabular}

Table V Axial Length and Masses for 7.5-hp Rating. Per-Unit Length Is Defined with Respect to the PolePitch Arc Length, and Mass Values are Per Unitized to Typical Design [see Fig. 5(d)]

\begin{tabular}{|l|c|c|c|c|}
\hline & $\mathrm{M}-1$ & $\mathrm{M}-2$ & $\mathrm{M}-3$ & Typ. \\
\hline Axial length [pu] & 2.08 & 2.33 & 2.73 & 2.85 \\
\hline PM mass [pu] & 0.93 & 1.05 & 1.25 & 1.00 \\
\hline Total mass [pu] & 0.75 & 0.81 & 0.92 & 1.00 \\
\hline
\end{tabular}




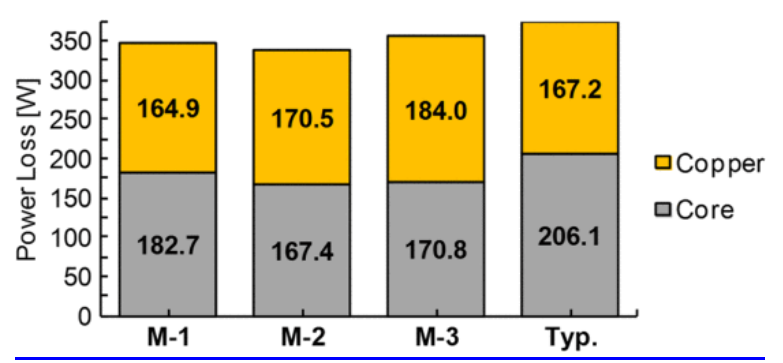

Fig. 8. Separation of loss components: Core loss (hysteresis and eddy) and copper winding loss for machines in Fig. 5.

\section{Discussion}

The optimization results presented in this paper show several design trends that may seem unconventional. First, the optimized machines have a significant reduction, of up to an order of magnitude, of both rated-load and open-circuit (cogging) torque ripples (see Fig. 6). This is while the induced voltage waveforms at both open-circuit and rated-load conditions are rich in their harmonic content, as can be observed from Fig. 7 and Table IV. In the optimized machines, the reduction in the torque ripple is achieved through a combination of design variables, namely, air-gap height, slot opening and tooth-tip shape (tip depth and tip angle), and the pole-arc length. In the optimized machines, these parameters result in a balance between the torque ripple produced by the back-EMF harmonics and the torque ripple resulting from the position-dependent variation of the stored magnetic energy as discussed in [14]. It should be noted that significant reduction of torque ripple levels in the optimized machines eliminates the need for stator and/or rotor skewing. The ripple-free operation of nonskewed optimized machines can be achieved with a well-tuned sine-wave currentregulated drive, capable of regulating/eliminating higher order current harmonics (5th and 7th) that may result from the back-EMF harmonics.

Second, the three optimized machines tend to have lower pole-arc coverage ( 0.62 average), resulting in higher PM flux concentration/focusing. As can be seen from Fig. 7, this results in an increase of the fundamental component of the induced voltage of the optimized machines. For example, for machine $M-1$, operating under open-circuit conditions, the induced voltage is increased by $22 \%$ in comparison to that for the machine that has a conventional pole-arc coverage of 0.78 .

\section{SECTION V. Case Study 2: High-Power PM Machines}

\section{A. Problem Statement}

In the four machines shown in Fig. 9, two stator cores, the fractional-slot with $q=0.5$ and the integerslot with $q=2$, and two rotor topologies, the SPM with radial magnetization and the generic IPM with single layer and uniform/parallel magnetization, are considered. In the fractional-slot topologies, the semiclosed slot opening was preferred to enable the generalization of results to a wide range of designs and ratings. For the larger machines, the magnetic equivalent of the tooth tip shown in Fig. 9(a) and (b) can be realized, for example, by using a magnetic wedge. In the design of the machines, the outside diameter, conductor current density, frequency, and number of poles are assumed to be constant and are summarized in Table VI. The outer diameter of the machine has been fixed to a value of $3 \mathrm{~m}$, while the slot fills corresponding to the integer-slot and fractional-slot 
topologies are equal to $35 \%$ and $40 \%$, respectively. The rare-earth magnet remanence and relative permeability are equal to $1.14 \mathrm{~T}$ and 1.1 , respectively. The steel assumed for the construction of both stator and rotor cores is M19-26Ga. The stator and rotor geometric design parameters and corresponding ranges used in the design process are summarized in Table VII. It should be noted that, in the case of the fractional-slot SPM and IPM machines [see Fig. 9(a) and (b)], seven and eight parameters are used for optimization, respectively, whereas in the case of the integer-slot SPM and IPM machines [see Fig. 9(c) and (d)], six and seven parameters are used, respectively.

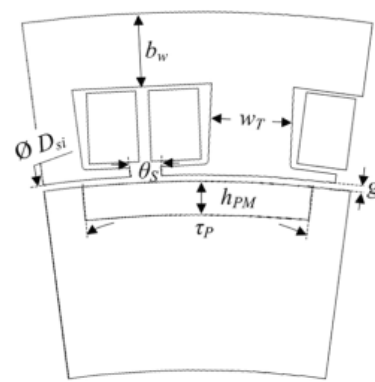

(a)

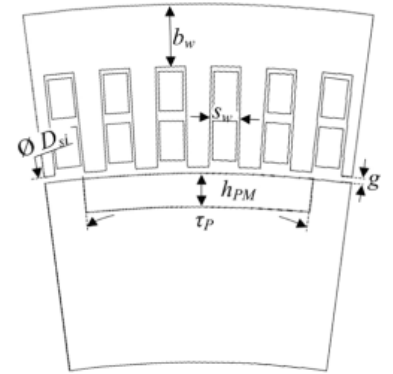

(c)

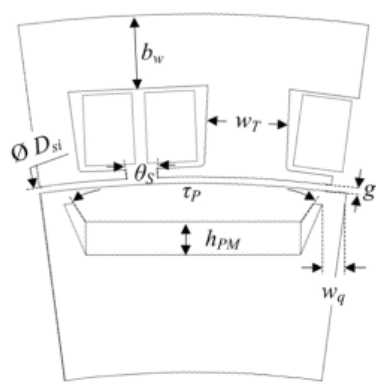

(b)

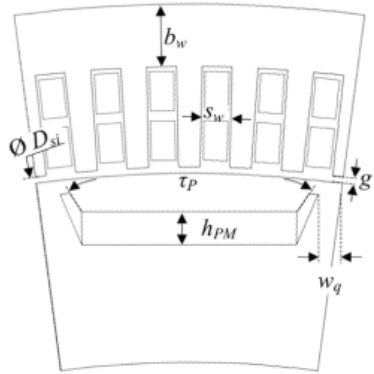

(d)

Fig. 9. Cross sections of four case-study machines, showing machine geometries. Due to the even periodicity, two poles of fractional-slot machines are modeled (a) and (b), and due to the odd periodicity, only a single pole of integer-slot machines is modeled (c) and (d).

Table VI Fixed Design Parameters for the Case Study 2 of Fig. 9

\begin{tabular}{|l|l|l|}
\hline & Fractional-slot & Integer-slot \\
\hline Number of slots & 36 & 144 \\
\hline Number of poles & 24 & 24 \\
\hline Rated frequency $[\mathrm{Hz}]$ & 30 & 30 \\
\hline Stator outer diameter, $D_{\text {so }}[\mathrm{mm}]$ & 3000 & 3000 \\
\hline Current density $A_{r m s} / \mathrm{mm}^{2}$ & 4.05 & 4.65 \\
\hline
\end{tabular}

Table VII Independent Design Variables and Limits for the Case Study 2 of Fig. 9

\begin{tabular}{|l|l|l|l|l|}
\hline & Fractional-slot & & Integer-slot & \\
\hline & $\min$ & $\max$ & $\min$ & $\max$ \\
\hline Stator inner diameter, $D_{S i}[\mathrm{~mm}]$ & 2500 & 2650 & 2500 & 2650 \\
\hline Air-gap height, $g[\mathrm{~mm}]$ & 4 & 10 & 4 & 10 \\
\hline Tooth width, $w_{T}[\mathrm{~mm}]$ & 50 & 125 & N/A & N/A \\
\hline Slot width, $w_{S}[\mathrm{~mm}]$ & $\mathrm{N} / \mathrm{A}$ & $\mathrm{N} / \mathrm{A}$ & 15 & 45 \\
\hline Back iron width, $w_{B}[\mathrm{~mm}]$ & 110 & 150 & 25 & 100 \\
\hline
\end{tabular}




\begin{tabular}{|l|l|l|l|l|}
\hline Slot opening, $\theta_{S}$ [deg. mech.] & 0.5 & 4 & N/A & N/A \\
\hline & SPM & & IPM & \\
\hline Pole arc angle, $\tau_{P}$ [deg. el.] & 110 & 170 & 110 & 150 \\
\hline PM height, $h_{P M}[\mathrm{~mm}]$ & 15 & 60 & 15 & 50 \\
\hline q-axis bridge, $w_{q}[\mathrm{~mm}]$ & 60 & 70 & N/A & N/A \\
\hline
\end{tabular}

An additional search of an optimum operating point (MTPA) was performed for every IPM candidate machine design. The four topologies shown in Fig. 9 were evaluated with 5000 candidate designs each, resulting in a total of 20000 candidate machine design evaluations. The total simulation time on a typical PC workstation utilizing a single core was equal to a very reasonable $124 \mathrm{~h}$. The detailed breakdown of model simulation times is provided in Table VIII. It should be noted that further reduction of the simulation time is achievable by evaluating CE-FEA candidate design models in parallel on multiple cores.

Table VIII Number of Magnetostatic Solutions and Computational Time Required for Evaluation of a Single Candidate Design (Including MTPA Search for IPMs)

\begin{tabular}{|l|l|l|l|}
\hline Fractional-slot SPM & & & \\
\hline CE-FEA & Solutions & Mesh density/Order & Computational Time [s] \\
\hline Detailed & 7 & 2991 el. / 2nd & 15.7 \\
\hline TOTAL & $1 \times$ Detailed & & 15.7 \\
\hline Fractional-slot 1PM & & & \\
\hline Reduced & 3 & 2588 el. / 1st & 2.5 \\
\hline Detailed & 7 & 2588 el. / 2nd & 16.1 \\
\hline TOTAL & $4 \times$ Reduced + I X Detailed & & 26.1 \\
\hline Integer-slot SPM & & & \\
\hline Detailed & 9 & 1963 el. / 2nd & 19.5 \\
\hline TOTAL & $1 \times$ Detailed & & 19.5 \\
\hline Integer-slot 1PM & & & \\
\hline Reduced & 3 & 1852 el. / 1st & 2.1 \\
\hline Detailed & 9 & 1852 el. / 2nd & 20.2 \\
\hline TOTAL & $4 \times$ Reduced + I X Detailed & & 28.6 \\
\hline
\end{tabular}

\section{B. Optimization Results}

The optimization results of the four machine topologies (see Fig. 9) are shown in Fig. 10. Shown in Fig. 10 are the objective function values, i.e., the electromagnetic torque per unit axial length and the torque ripple at rated load, subject to parameter constraints provided in Table VII. As mentioned before, the outside stator diameter is fixed to $3 \mathrm{~m}$; hence, here, the specific torque is defined as the torque per unit axial length of the machine. The objective functions corresponding to all candidate designs (20 000) are shown in Fig. 10. Also shown in Fig. 10 are the Pareto-optimal sets corresponding to every machine topology of Fig. 9. 


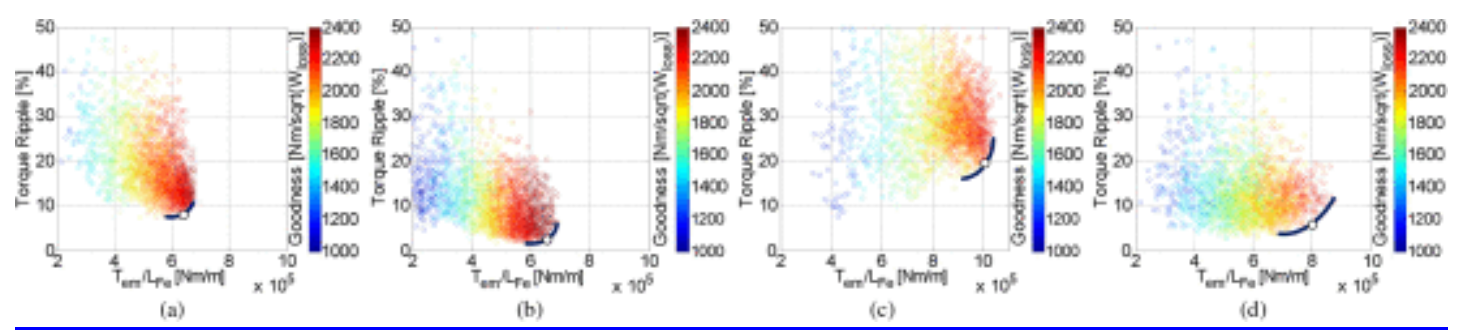

Fig. 10. Design objectives showing the following: Design objectives of all candidate designs, Pareto-optimal sets after 50 generations, and the selected optimal machines. (a) F-SPM. (b) F-IPM. (c) I-SPM. (d) I-IPM.

Depicted in Fig. 11 are the evolutions of the design parameters for the fractional-slot embedded SPM machine during the course of optimization. From Fig. 11, it should be noted that, in the multi-objective optimization that results in the distributed Pareto-optimal sets, such as the ones shown in Fig. 10, the design parameters are not guaranteed to converge to constant values. This is in contrast to singleobjective optimization studies where all design parameters are guaranteed to converge to constant optimal values corresponding to a single optimal machine design. As can be seen from Fig. 11, design variables converge to a bounded region of parameter values which corresponds to designs in the Pareto-optimal set.

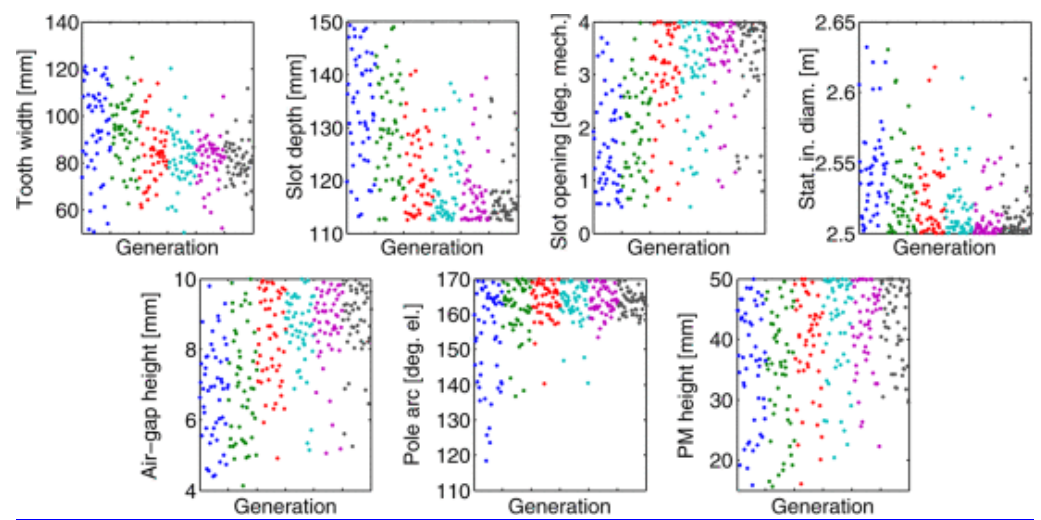

Fig. 11. Evolution of design parameters of fractional-slot embedded SPM motor during the 50 generations (only the (blue) 1st, (green) 10th, (red) 20th, (cyan) 30th, (purple) 40th, and (black) 50th generations are plotted). Every point represents a candidate design parameter in the corresponding generation. Variables are not expected to converge to constant values in a multi-objective optimization.

As can be observed from the results of Fig. 10, the two objectives considered in the optimization study are conflicting. This means that the choice of an optimal machine design involves evaluating tradeoffs between the designs in the Pareto-optimal set. From Fig. 10, a large variability of the specific torque, torque ripple, and "goodness" should be noted. Comparing integer-slot and fractional-slot machines, one should notice a significant increase of the specific torque for the machines with integer-slot stators. This can be explained by the fact that the fractional-slot topology used in this work has a low fundamental winding factor of 0.866 . Also, as expected, fractional-slot machine designs have lower torque ripple values when compared to the integer-slot machines. On the other hand, comparison of the "goodness" of the fractional and integer-slot machines may be affected by several factors not fully accounted for in the current analysis. More specifically, fractional-slot winding machine designs may result in increased eddy-current losses in the PMs and in the stator windings due to the high harmonic 
content of the armature MMF. Another factor that may affect the comparison of the loss performance of the two winding topologies is the active axial length, which affects the ratio of end winding losses to the total machine losses.

Four optimal designs corresponding to every motor topology are selected from the four optimal sets shown in Fig. 10. Namely, these designs are the fractional-slot SPM (F-SPM), F-IPM, integer-slot SPM (ISPM), and I-IPM.

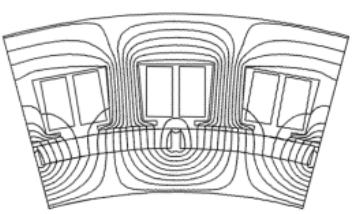

(a)

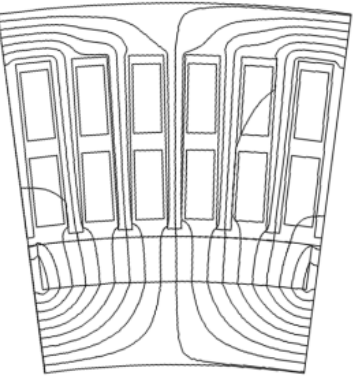

(c)

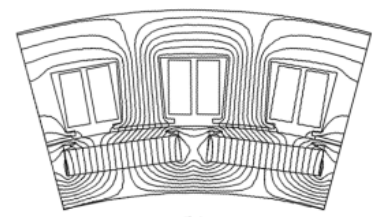

(b)

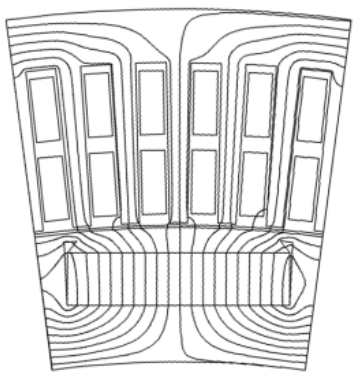

(d)

Fig. 12. Cross sections of four optimal machines, showing machine geometries and open-circuit flux distributions. Due to the even periodicity, two poles of fractional-slot machines are modeled (a) and (b), and due to the odd periodicity, only a single pole of integer-slot machines is modeled (c) and (d). The combination of (a) and (b) has a different scale with the combination of (c) and (d).

The designs are selected based on the best compromise between the specific torque and the torque ripple corresponding to every Pareto-set in Fig. 10. The cross sections corresponding to the four optimal designs and the corresponding parameters are provided in Fig. 12 and Table IX.

From Fig. 12(b) and (d), one should note that the nonmagnetic space at the end of the magnets was not geometrically correlated as a dependent variable of the magnet arc or length facing the air gap. It was expected that the optimization algorithm would push the magnet to the maximum allowable dimensions to a configuration that has no space at the end of the magnet as shown in Fig. 9(b) and (d).

Table IX Optimal Design Parameters Corresponding to Machines Shown in Fig. 12

\begin{tabular}{|l|l|l|l|l|}
\hline & F-SPM & F-IPM & I-SPM & I-IPM \\
\hline Stator inner diameter, $D_{S i}[\mathrm{~mm}]$ & 2500 & 2505 & 2501 & 2515 \\
\hline Air-gap height, $g[\mathrm{~mm}]$ & 9.0 & 7.1 & 8.8 & 5.6 \\
\hline Tooth width, $w_{T}[\mathrm{~mm}]$ & 76.6 & 96.7 & N/A & N/A \\
\hline Slot width, $w_{S}[\mathrm{~mm}]$ & N/A & N/A & 38.9 & 34.3 \\
\hline Back iron width, $w_{B}[\mathrm{~mm}]$ & 113.0 & 113.1 & 57.5 & 61.0 \\
\hline Slot opening, $\theta_{S}[$ deg. mech.] & 4.0 & 3.85 & N/A & N/A \\
\hline Axial length, $l_{F e}[\mathrm{~mm}]$ & 1953 & 2028 & 1276 & 1576 \\
\hline Pole arc angle, $\tau_{P}[$ deg. el.] & 165.9 & 110.1 & 169.3 & 128.1 \\
\hline PM height, $h_{P M}[\mathrm{~mm}]$ & 49.7 & 56.4 & 50.5 & 59.7 \\
\hline PM height, $h_{w}[\mathrm{~mm}]$ & N/A & 251.2 & N/A & 252.5 \\
\hline
\end{tabular}




\begin{tabular}{|l|l|l|l|l|}
\hline q-axis bridge, $w_{q}[\mathrm{~mm}]$ & N/A & 68.9 & N/A & 65.2 \\
\hline
\end{tabular}

Depicted in Fig. 13(a) and (b) are the torque profiles corresponding to the four machines operating at rated-load (MTPA) and open-circuit (cogging torque) conditions, respectively. As mentioned previously, a significant increase of the specific torque is observed for the integer-slot machines (I-SPM and I-IPM). On the other hand, a significant improvement of the torque ripple is achieved with fractional-slot designs (F-SPM and F-IPM). Ripple-free operation of the optimized F-IPM machine, at both rated-load and open-circuit conditions, should be noted. The torque profiles shown in Fig. 13 were verified with a detailed FEA that employs the Maxwell stress tensor and second-order finite elements [16].

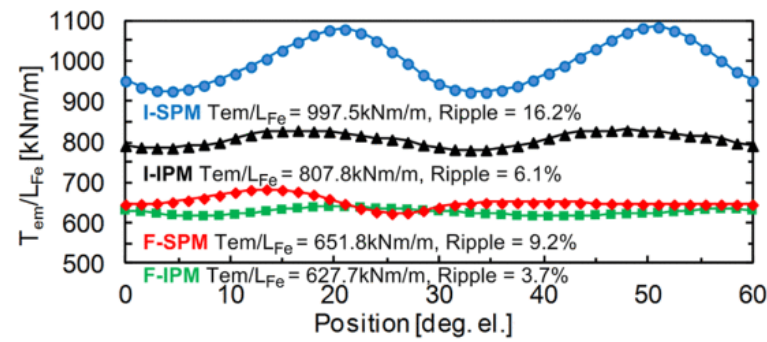

(a)

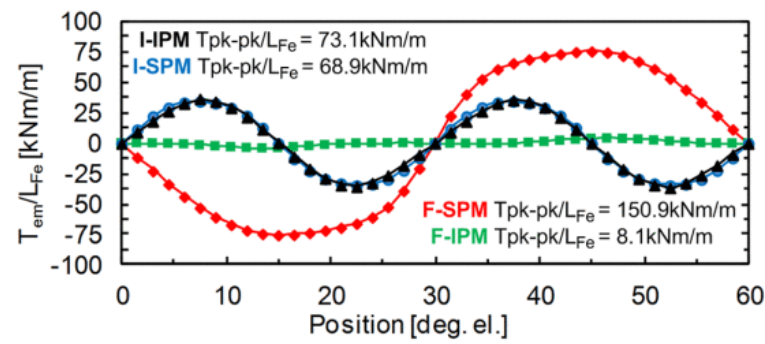

(b)

Fig. 13. Electromagnetic torque for machines of Fig. 12 (red: F-SPM; green: F-IPM; blue: I-SPM; black: I-IPM) supplied by a current-regulated sine-wave drive (purely sinusoidal currents are assumed). Torque profiles are verified/obtained using detailed FEA with Maxwell stress tensor and second-order elements.

\section{Design to Achieve Desired Rating of $20 \mathrm{MW}$ and $150 \mathrm{r} / \mathrm{min}$}

In this section, four optimized machines (F-SPM, F-IPM, I-SPM, and I-IPM) were scaled axially to achieve the desired 20-MW output rating. The corresponding axial lengths for the four machines are provided in Table IX. Also depicted in Table $X$ are the PM and total machine masses corresponding to the four optimal designs. As expected, I-SPM machine [see Fig. 12(c)], having the highest specific torque [see Fig. 13(a)], results in the shortest and lightest machine that utilizes the least PM material for the given rating. The PM mass and the total active material mass (magnet, copper, and iron) of the I-SPM design are 3440 and $63467 \mathrm{~kg}$, respectively.

Table X PM Mass and Total Active Machine Mass. Per-Unit PM Mass of I-SPM Optimal Design with PM Mass of $3440 \mathrm{~kg}$. Per-Unit Total Mass of I-SPM Optimal Design with Total Active Mass of $63467 \mathrm{~kg}$

\begin{tabular}{|l|l|l|l|l|}
\hline & F-SPM & F-IPM & I-SPM & I-IPM \\
\hline PM mass [pu] & 1.49 & 1.58 & 1.00 & 1.24 \\
\hline Total mass[pu] & 1.57 & 1.64 & 1.00 & 1.25 \\
\hline
\end{tabular}




\section{Discussion}

The optimized machines presented in this paper aim at improving torque production quality while minimizing active machine mass and losses. These objectives are oftentimes conflicting as can be observed from Fig. 10. In other words, selecting a design with high specific torque usually results in a machine with high torque ripple. In addition, selecting a design with high specific torque may result in a machine having a significantly shorter axial length, hence increasing the proportion of end winding losses in the overall total machine loss and potentially reducing the efficiency. In addition, machines with high specific torque result in smaller machines with less surface area to dissipate the losses, hence potentially requiring more sophisticated thermal management.

As shown in this paper, accurate comparison of different machine topologies should be performed on the basis of comparing their respective Pareto-optimal sets. In this approach, the variability of design objectives due to design parameters is eliminated. Hence, only optimal machines corresponding to every topology (number of slots/poles, magnet arrangement, etc.) are compared. For example, considering the fractional slot with SPM [see Fig. 10(a)] and IPM [see Fig. 10(b)] arrangements, there exists a large number of suboptimal designs that result in similar design objectives. However, comparing the Pareto-optimal sets, it is clear that the IPM design outperforms the SPM design in terms of both the specific torque and torque ripple performance.

\section{SECTION VI. Conclusion}

In this paper, a comprehensive FEA-based multi-objective design optimization method for PM ac machines has been presented. Two examples demonstrate that this approach, which is based on the recently developed CE-FEA technique, is well suited for large-scale design synthesis and optimization. The developed method relies solely on FEA for the calculation of relevant performance information using modest PC-based computational facilities and practically eliminates the need for using less accurate analytical and lumped parameter equivalent circuit models in automated electric machine design.

For the first case study of an F-IPM motor rated at $7.5 \mathrm{hp}$, a total of 11 stator and rotor independent design variables have been simultaneously optimized to satisfy multiple design criteria. The total simulation time for 10000 designs, which included the search of the MTPA condition for every candidate design, was $52 \mathrm{~h}$ on a typical PC-based workstation. In the second case study, four integral megawatt direct-drive machines, namely, fractional-slot embedded SPM, fractional-slot IPM, integerslot embedded SPM, and integer-slot IPM, with a total of 20000 candidate machine designs have been evaluated in $124 \mathrm{~h}$ with the same hardware and software setup.

Results presented in this paper highlight the conflicting nature of typical design objectives (torque density, torque ripple, and efficiency). In addition, results presented in this paper show that torquedense and low-torque-ripple motors can be designed using automated design tools such as the one introduced by the authors.

The Pareto-optimal design sets derived through a DE algorithm can serve as a basis for quantitatively analyzing the tradeoffs specific to multi-objective optimization. The first case study has interestingly showed that an optimal design with high efficiency and virtually zero torque ripple can be achieved even for an F-IPM topology with three slots per pole pair. In the second case study, different machine 
topologies were systematically compared in terms of specific torque output, torque ripple, and cost, leading to an optimal selection.

\section{ACKNOWLEDGMENT}

The engineering support of A. O. Smith Corporation is gratefully acknowledged. The authors would also like to thank ANSYS Inc. for providing access to Maxwell 2D/3D FEA simulation software.

\section{References}

1. Y. Duan and D. M. Ionel, "A review of recent developments in electrical machine design optimization methods with a permanent-magnet synchronous motor benchmark study", IEEE Trans. Ind. Appl., vol. 49, no. 3, pp. 1268-1275, May/Jun. 2013.

2. W. Jiang, T. M. Jahns, T. A. Lipo, W. Taylor and Y. Suziki, "Machine design optimization based on finite element analysis in a high-throughput computing environment", Proc. IEEE ECCE, pp. 869876, 2012-Sep.

3. F. Parasiliti, M. Villani, S. Lucidi and F. Rinaldi, "Finite-element-based multiobjective design optimization procedure of interior permanent magnet synchronous motors for wide constantpower region operation", IEEE Trans. Ind. Electron., vol. 59, no. 6, pp. 2503-2514, Jun. 2012.

4. G. Pellegrino and F. Cupertino, "FEA-based multi-objective optimization of IPM motor design including rotor losses", Proc. IEEE ECCE, pp. 3659-3666, 2010-Sep.

5. A. Arkadan, M. ElBsat and M. Mneimneh, "Particle swarm design optimization of ALA rotor SynRM for traction applications", IEEE Trans. Magn., vol. 45, no. 3, pp. 956-959, Mar. 2009.

6. S. Giurgea, D. Fodorean, G. Cirricione, A. Miraoui and M. Cirrincione, "Multimodel optimization based on the response surface of the reduced FEM simulation model with application to a PMSM", IEEE Trans. Magn., vol. 46, no. 2, pp. 2153-2157, Sep. 2008.

7. A. Sarikhani and O. A. Mohammed, "HIL-based finite-element design optimization process for the computational prototyping of electric motor drives", IEEE Trans. Energy Conversion, vol. 27, no. 3, pp. 737-746, Sep. 2012.

8. W. Ouyang, D. Zarko and T. A. Lipo, "Permanent magnet machine design practice and optimization", Conf. Rec. 41st IEEE IAS Annu. Meeting, vol. 4, pp. 1905-1911, 2006-Oct.

9. D. A. Staton, R. P. Deodhar, W. L. Soong and T. J. E. Miller, "Torque prediction using the flux-MMF diagram in ac dc and reluctance motors", IEEE Trans. Ind. Appl., vol. 32, no. 1, pp. 180-188, Jan./Feb. 1996.

10. D. M. Ionel, M. Popescu, M. I. McGilp, T. J. E. Miller and S. J. Dellinger, "Assessment of torque components in brushless permanent-magnet machines through numerical analysis of the electromagnetic field", IEEE Trans. Ind. Appl., vol. 41, no. 5, pp. 1149-1158, Sep./Oct. 2005.

11. T. J. E. Miller, M. Popescu, C. Cossar, M. I. McGilp, M. Olaru, A. Davies, et al., "Embedded finiteelement solver for computation of brushless permanent-magnet motors", IEEE Trans. Ind. Appl., vol. 44, no. 4, pp. 1124-1133, Jul./Aug. 2008.

12. F. A. Fouad, T. W. Nehl and N. A. Demerdash, "Magnetic field modeling of permanent magnet type electronically operated synchronous machines using finite elements", IEEE Trans. Power App. Syst., vol. PAS-100, no. 9, pp. 4125-4135, Sep. 1981. 
13. D. M. Ionel and M. Popescu, "Finite-element surrogate model for electric machines with revolving field-application to IPM motors", IEEE Trans. Ind. Appl., vol. 46, no. 6, pp. 2424-2433, Nov./Dec. 2010.

14. G. Y. Sizov, D. M. lonel and N. A. O. Demerdash, "Modeling and parametric design of permanentmagnet ac machines using computationally efficient finite-element analysis", IEEE Trans. Ind. Electron., vol. 59, no. 6, pp. 2403-2413, Jun. 2012.

15. N. Bianchi and L. Alberti, "MMF harmonics effect on the embedded FE analytical computation of PM motors", IEEE Trans. Ind. Appl., vol. 46, no. 2, pp. 812-820, Mar./Apr. 2010.

16. ANSYS Maxwell.

17. K. V. Price, R. M. Storn and J. A. Lampinen, Differential Evolution-A Practical Approach to Global Optimization, Germany, Berlin:Springer-Verlag, 2005.

18. F. Deng and T. W. Nehl, "Analytical modeling of eddy-current losses caused by pulse-widthmodulation switching in permanent-magnet brushless direct-current motors", IEEE Trans. Magn., vol. 34, no. 5, pp. 3728-3736, Sep. 1998.

19. N. A. Demerdash and H. B. Hamilton, "Effect of rotor asymmetry on field forms and eddy current losses in stator conductors due to radial flux", IEEE Trans. Power App. Syst., vol. PAS-91, no. 5, pp. 1999-2010, Sep. 1972.

20. P. B. Reddy, T. M. Jahns and T. P. Bohn, "Modeling and analysis of proximity losses in high-speed surface permanent magnet machines with concentrated windings", Proc. IEEE ECCE, pp. 9961003, 2010-Sept.

21. A. Boglietti, A. Cavagnino and M. Lazzari, "Fast method for the iron loss prediction in inverter-fed induction motors", IEEE Trans. Ind. Appl., vol. 46, no. 2, pp. 806-811, Mar./Apr. 2010.

22. D. Lin, P. Zhou and Q. M. Chen, "The effects of steel lamination core losses on transient magnetic fields using $\backslash \$$ hbox $\{T\}$ - omega \\$ method", Proc. IEEE VPPC, pp. 1-4, 2008-Sep.

23. K. Deb, Multi-Objective Optimization Using Evolutionary Algorithms, USA, NY, New York:Wiley, 2001. 